\title{
DESEMPENHO DE HERBICIDAS PRÉ-EMERGENTES NO CONTROLE DE CAPIM-ARROZ E NABO NA CULTURA DA SOJA
}

\author{
Fernanda Cassiane Caratti ${ }^{1}$; Thaís D’Avila Rosa ${ }^{1}$; Luiza Piccinini Silveira ${ }^{1}$; Joice \\ Fernanda Bonow ${ }^{1}$; Daniele Brandstetter Rodrigues ${ }^{2}$ \\ 1 Mestrandas do Departamento de Fitossanidade, Universidade Federal de \\ Pelotas (nandacaratti@yahoo.com.br) Rio grande do sul - Brasil \\ 2 Doutorando do Departamento de Fitotecnia, Universidade Federal de Pelotas \\ (thais.d.rosa@hotmail.com) Rio Grande do Sul- Brasil \\ Recebido em: 08/09/2015 - Aprovado em: 14/11/2015 - Publicado em: 01/12/2015 \\ DOI: http://dx.doi.org/10.18677/Enciclopedia_Biosfera_2015_127
}

\begin{abstract}
A competição com plantas daninhas em lavouras de soja é um dos principais fatores que comprometem a produtividade da cultura. Herbicidas pré-emergentes são excelentes ferramentas no manejo das plantas daninhas, pois permitem controle eficiente durante 0 crescimento inicial da cultura. Objetivou-se, investigar 0 desempenho de herbicidas pré-emergentes na cultura da soja. O experimento foi realizado em casa-de-vegetação da Faculdade de Agronomia Eliseu Maciel Universidade Federal de Pelotas nos meses de outubro a novembro de 2014 . O experimento foi arranjado em delineamento inteiramente casualizado, as unidades experimentais foram constituídas por vasos onde foi semeado a cultivar de soja Potência e infestados com capim-arroz e nabo, os tratamentos foram aplicação em pré-emergência de clomazone, pendimethalin, diclosulam, s-metolachlor, sulfentrazone, sem aplicação e sem infestação, e sem aplicação com infestação. As avaliações foram realizadas aos 7, 14, 21 e 28 DAE, sendo estatura das plantas de soja e fitotoxicidade, e controle das plantas daninhas, bem como a massa da matéria seca da parte aérea e o índice de clorofila aos 28 DAE. A cultivar de soja Potência apresentou redução no comprimento da parte aérea no tratamento com pendimethalin nas primeiras épocas de avaliação, porém houve superação a partir dos 21 DAE, equivalendo-se aos demais. Os herbicidas diclosulam e sulfentrazone apresentaram maior fitotoxicidade na soja aos 28 DAE. O melhor controle de nabo foi obtido com pendimethalin e clomazone e para o capim-arroz o melhor controle também foi observado para os referidos herbicidas acrescendo s-metolachlor. Dos herbicidas pré-emergentes avaliados o sulfentrazone apresentou maior fitotoxicidade a cultura da soja bem como um menor controle das plantas daninhas.
\end{abstract}

8uPALAVRAS-CHAVE: Glycine max, controle, fitotoxicidade, planta daninha, residual. 


\title{
PERFORMANCE OF PRE-EMERGENCE HERBICIDES IN BARNYARDGRASS CONTROL AND TURNIP IN SOYBEAN
}

\begin{abstract}
Competition from weeds in soybean crops is one of the main factors that compromise the productivity of the crop. Herbicides pre-emergence are excellent tools in the management of weeds as they allow efficient control during the early growth of the crop. The objective of investigating the performance of pre-emergence herbicides in soybean. The experiment was carried out in a greenhouse at the Faculty of Agronomy Eliseu Maciel - Federal University of Pelotas, in the months from October to November 2014. The experiment was arranged in a randomized design, where the experimental unit consisted of vessels in which was sown in soybean Potência and infested with barnyardgrass and turnip, treatments were applied pre-emergence of clomazone, pendimethalin, diclosulam, s-metolachlor, sulfentrazone, without application and without infestation, and without application infestation. The evaluations were performed at 7, 14, 21 and $28 \mathrm{DAE}$, and stature of soybean and phytotoxicity Plant and weed control, and the dry mass and the chlorophyll content at 28 DAE. The soybean cultivar Potência decreased in shoot length of treatment with pendimethalin in the first evaluation times, but there was overcoming from 21 DAE, equivalent to the others. Herbicides diclosulam and sulfentrazone showed higher hytotoxicity in soybean at 28 DAE. The best turnip control was obtained with pendimethalin and clomazone, for barnyardgrass better control was also observed for those adding s-metolachlor herbicides. Pre-emergence herbicides evaluated Sulfentrazone presented Soy culture most phytotoxicity as well as a minor weed control.
\end{abstract}

KEYWORDS: control, Glycine max, phytotoxicity, residual, weed.

\section{INTRODUÇÃO}

O cultivo da soja (Glycine max) é dependente do uso de herbicidas para o controle de plantas daninhas, evitando-se assim perdas por competição. Um dos motivos que levam a esta dependência é o fato do controle químico proporcionar o manejo mais simples e eficaz das espécies indesejadas. Além disso, o uso de herbicidas pré-emergentes com efeito residual prolongado é determinante na eficiência do controle de plantas daninhas durante o período crítico de competição (DAN et al., 2010).

A mobilidade dos herbicidas no solo é dependente de alguns fatores como material de origem do solo, minerais da fração argila, quantidade de matéria orgânica, bem como das condições climáticas no momento da aplicação (WEBER et al., 2004). Assim, o conhecimento das características do solo, da seletividade, persistência e modo de ação dos herbicidas são indispensáveis para permitir o uso correto dos mesmos (MONQUERO, 2010).

A persistência da molécula do herbicida no solo é dependente de diversos fatores, sendo de suma importância considerar algumas características do solo, como textura, umidade, teor de matéria orgânica quando do uso de herbicidas de solo, visto que estes fatores possuem influência marcante na dinâmica dos herbicidas no ambiente. O tipo de solo é determinante em relação ao potencial de lixiviação e a capacidade de adsorção do produto, sendo que as moléculas de herbicidas são mais adsorvidas em solos argilosos, e mais suscetíveis a lixiviação em solos arenosos (MONQUERO et al., 2010). Ainda conforme autores, alguns 
herbicidas possuem elevada capacidade adsortiva que é dependente do conteúdo de matéria orgânica do solo, sendo maior a adsorção quanto mais alto for o teor de matéria orgânica, e assim menor a lixiviação.

A cultura da soja, se conduzida atendendo as boas práticas de manejo, pode chegar ao máximo potencial produtivo. Para tanto é necessário que se inclua no manejo, a fertilidade necessária, a adequada época de semeadura cujas importâncias são fundamentais na produtividade da cultura. Outro aspecto importante no sistema de produção é o manejo de plantas daninhas, doenças e pragas. Em relação a ocorrência de plantas daninhas o maior prejuízo está na redução do rendimento de grão das culturas, dada a competição por água, luz e nutrientes que se estabelece (VIDAL \& MEROTTO 2001).

De fato o controle das plantas daninhas é extremamente importante, permitindo que a cultura expresse seu potencial produtivo estando livre de competição por recursos como água, luz e nutrientes. Os efeitos negativos da interferência das plantas daninhas sobre a cultura dependem de alguns fatores, como a espécies infestante, da sua densidade e distribuição na área e do espaçamento da cultura, além do período de convivência entre a cultura e planta daninha (VELHO et al., 2012). Com base nisso é de suma importância à adoção de estratégias que permitam o controle de plantas daninhas nas lavouras, como a aplicação de herbicidas em pré e pós-emergência, prática esta comum adotada em diferentes sistemas produtivos (VARGAS et al., 2013).

O sistema de produção de soja RR favoreceu o aumento da frequência de espécies de plantas daninhas com tolerância natural ao glifosato ou controladas apenas parcialmente pelo produto (CORREIA \& DURIGAN, 2010), visto que esta molécula foi ao longo do tempo selecionando biótipos resistentes ao seu mecanismo de ação. Diante disso uso de herbicidas com residual, seletivos à cultura parecem ser uma ferramenta importante na estratégia de manejo das plantas daninhas resistentes.

O objetivo deste trabalho foi avaliar o desempenho de herbicidas préemergentes na cultura da soja quanto à seletividade e o controle das plantas daninhas capim-arroz e nabo, de ocorrência típica em lavouras de soja na várzea e sequeiro respectivamente.

\section{MATERIAL E MÉTODOS}

Foi conduzido um experimento em casa-de-vegetação da Faculdade de Agronomia Eliseu Maciel, Universidade Federal de Pelotas/UFPEL, no período de outubro a novembro de 2014. Os tratamentos foram aplicação em pré-emergência de clomazone (1.250 g i.a. ha $\left.{ }^{-1}\right)$, pendimethalin (1.000 g i.a. ha $\left.{ }^{-1}\right)$, diclosulam (35 g i.a. ha $\left.{ }^{-1}\right)$, s-metolachlor $\left(2.880 \mathrm{~g}^{-}\right.$i.a. ha $\left.{ }^{-1}\right)$, sulfentrazone $\left(600 \mathrm{~g}\right.$ i.a. ha $\left.{ }^{-1}\right)$, sem aplicação e sem infestação, e sem aplicação com infestação.

As unidades experimentais foram constituídas por vasos de 8 litros de capacidade, preenchidos com solo com adubação corrigida de $250 \mathrm{~kg} / \mathrm{ha}(5-20-20)$, cujas características físico-químicas encontram-se na Tabela 1. Estes foram infestados pelas plantas daninhas capim-arroz e nabo, em seguida semeou-se a soja e aplicou-se os herbicidas. 
TABELA 1. Características físico-químicas da camada de $0-0,2 \mathrm{~m}$ de profundidade do solo. FAEM/UFPel - Capão do Leão, RS, 2014.

\begin{tabular}{|c|c|c|c|}
\hline p. & Argila M.O & Fósforo Potássio & Cálcio Magnésio Alumínio \\
\hline 5,4 & $15^{1,10}$ & $9,4-61$ & 0,9 \\
\hline
\end{tabular}

A aplicação dos tratamentos foi realizada com pulverizador costal pressurizado com $\mathrm{CO}_{2}$, a pressão constante de 3 bar, equipado com 3 pontas tipo leque com indução a ar, Teejet Al 11002 distanciados entre si em 0,50 m, e barra de aplicação de $1,5 \mathrm{~m}$ de largura, com volume de calda de $165 \mathrm{~L} \mathrm{ha}^{-1}$.

Aos 7, 14, 21, 28 dias após a emergência (DAE) foi avaliado o controle das plantas daninhas efetuado por escala percentual, estimando os valores visualmente atribuindo-se notas de 0 a $100 \%$, onde zero corresponde a ausência de controle e $100 \%$ corresponde à morte completa das plantas ou controle total. As avaliações de fitotoxicidade nas plantas de soja foram realizadas aos 7, 14 e 21 e 28 dias após a emergência (DAE), atribuindo notas entre 0 (referente a ausência de lesões) e 100 (morte das plântulas). Nessas referidas épocas de avaliação também se determinou a estatura das plantas de soja, medindo-se no colmo principal, a distância do nível do solo até o ápice, com auxílio de uma régua milimetrada.

Aos $28 \mathrm{DAE}$, posterior a avaliação dos comprimentos foi verificada a massa da matéria seca da parte aérea no qual as plantas foram mantidas em sacos de papel, em estufa à $60^{\circ} \mathrm{C}$, até a obtenção de massa constante, pesada em balança de precisão $(0,001 \mathrm{~g})$ e o valor obtido pela soma de cada repetição foi dividido pelo número de plantas utilizadas. Os resultados foram expressos em gramas planta ${ }^{-1}$.

Os resultados obtidos para as variáveis foram submetidos à análise de variância, e havendo significância pelo teste $F(P<0,05)$, as médias dos tratamentos foram submetidas a comparação pelo teste de Tukey $(P<0,05)$.

\section{RESULTADOS E DISCUSSÃO}

O comprimento da parte aérea aos 7 DAE apresentou diferença estatística apenas para o tratamento pendimethalin, quando comparado aos demais, não sendo observada diferença para esta data entre os demais tratamentos (Tabela 2).

Para a avaliação realizada aos 14 DAE observou-se que o menor crescimento da parte aérea foi obtido novamente para o pendimethalin. No entanto não se observou diferença entre os tratamentos pendimethalin e sulfentrazone. $O$ sulfentrazone não apresentou diferença entre os demais exceto quando comparado a testemunha limpa a qual apresentou crescimento superior em relação aos demais, porém não diferiu dos resultados observados para a testemunha suja, s-metolachlor, clomazone e diclosulam (Tabela 2).

Para as avaliações realizadas aos 21 e 28 DAE, não se observou diferença estatística entre os tratamentos para a variável comprimento da parte aérea, entendendo-se que houve uma compensação pelas plantas de soja, o que permitiu que estas recuperassem o estresse sofrido no início do seu desenvolvimento (Tabela 2). 
TABELA 2. Comprimento da parte aérea de plantas de soja aos 7, 14, 21 e 28 DAE. FAEM/UFPel- Capão do Leão, RS, 2014.

\begin{tabular}{lllll}
\hline Tratamentos & \multicolumn{4}{c}{ Comprimento Parte Aérea } \\
\hline & $7 \mathrm{DAE}$ & $14 \mathrm{DAE}$ & $21 \mathrm{DAE}$ & $28 \mathrm{DAE}$ \\
\cline { 2 - 5 } Testemunha limpa & $13,05 \mathrm{a}$ & $14,53 \mathrm{a}$ & $14,12^{\mathrm{ns}}$ & $17,05^{\mathrm{ns}}$ \\
testemunha suja & $12,43 \mathrm{a}$ & $14,45 \mathrm{ab}$ & 15,75 & 15,31 \\
S-metolachlor & $11,20 \mathrm{a}$ & $13,30 \mathrm{ab}$ & 14,80 & 15,32 \\
Clomazone & $11,69 \mathrm{a}$ & $13,27 \mathrm{ab}$ & 13,47 & 15,87 \\
Diclosulam & $11,76 \mathrm{a}$ & $11,64 \mathrm{ab}$ & 14,15 & 16,15 \\
Sulfentrazone & $10,68 \mathrm{a}$ & $10,66 \mathrm{bc}$ & 13,40 & 16,73 \\
Pendimethalin & $6,72 \mathrm{~b}$ & $9,11 \mathrm{c}$ & 11,27 & 14,71 \\
\hline CV $(\%)$ & 13,62 & 13,30 & 15,21 & 30,59 \\
\hline
\end{tabular}

Médias com letras minúsculas distintas na coluna diferem pelo teste Tukey $(p \leq 0,05)$.

${ }^{\text {ns }}$ Não significativo.

Para a fitotoxicidade avaliada na soja observou-se que aos 7 DAE o herbicida pendimethalin fui superior, porém não diferiu do sulfentrazone e nem do smetolachlor. Os resultados persistiram aos $14 \mathrm{DAE}$, mas houve uma redução nos níveis de fitotoxicidade se comparado ao observado na primeira época de avaliação, exceto para o Diclosulam que manteve o nível, tornando-se estatisticamente igual ao resultado observado para pendimenthalin, sulfentrazone e s-metolachlor. Os tratamentos com Diclosulam e sulfentrazone foram os que apresentaram maior fitotoxicidade na avaliação aos 28 DAE, mostrando-se estatisticamente superiores aos demais (Tabela 3 ).

TABELA 3. Avaliação de fitotoxicidade de plantas de soja aos 7, 14, 21 e 28 DAE. FAEM/UFPel- Capão do Leão, RS, 2014.

\begin{tabular}{|c|c|c|c|c|c|c|c|c|}
\hline \multirow[t]{2}{*}{ Tratamentos } & \multicolumn{8}{|c|}{ Fitotoxicidade (\%) } \\
\hline & \multicolumn{2}{|c|}{$7 \mathrm{DAE}$} & \multicolumn{2}{|c|}{$14 \mathrm{DAE}$} & \multicolumn{2}{|c|}{$21 \mathrm{DAE}$} & \multicolumn{2}{|l|}{$28 \mathrm{DAE}$} \\
\hline Testemunha limpa & 0,0 & $\mathrm{c}^{1}$ & 0,0 & $\mathrm{c}$ & 0,0 & $\mathrm{~b}$ & 0,0 & C \\
\hline Testemunha suja & & c & 0,0 & c & 0,0 & b & 0,0 & $\mathrm{c}$ \\
\hline S-metolachlor & 37,5 & $a b$ & 28,75 & $a b$ & 17,50 & $a b$ & 13,5 & b \\
\hline Clomazone & & $\mathrm{c}$ & 10,00 & bc & 16,25 & $a b$ & 11,5 & b \\
\hline Diclosulam & 32,5 & b & 32,50 & $a b$ & 23,75 & $a b$ & 26,5 & a \\
\hline Sulfentrazone & 36,5 & $a b$ & 32,50 & $a b$ & 31,25 & a & 30,0 & a \\
\hline Pendimenthalin & 63,5 & a & 46,25 & a & 15,00 & $a b$ & 8,75 & bc \\
\hline CV (\%) & 54,56 & & 53,20 & & 71,84 & & 30,59 & \\
\hline
\end{tabular}

${ }^{1}$ Médias com letras minúsculas distintas na coluna diferem pelo teste Tukey $(p \leq 0,05)$.

Houve diferença para no controle de nabo forrageiro para os herbicidas testados aos 7, 14, 21 e 28 DAE (Tabela 4). Os herbicidas clomazone e pendimenthalin foram os tratamentos que apresentaram os maiores índices de controle para a espécie em todas as avaliações, seguido dos tratamentos com smetolachlor e sulfentrazone. O herbicida Diclosulam foi o tratamento que apresentou baixos níveis de controle para o nabo, sendo que aos 21 e $28 \mathrm{DAE}$, o herbicida não diferiu das testemunhas limpa e suja. 
TABELA 4. Controle de nabo forrageiro aos 7, 14, 21 e 28 DAE. FAEM/UFPelCapão do Leão, RS, 2014.

\begin{tabular}{llllllll}
\hline \multirow{2}{*}{ Tratamentos } & \multicolumn{6}{c}{ Controle Nabo Forrageiro } \\
\cline { 2 - 8 } & $7 \mathrm{DAE}$ & \multicolumn{1}{c}{ 14 DAE } & $21 \mathrm{DAE}$ & $28 \mathrm{DAE}$ \\
\hline Testemunha limpa & $0,0 \mathrm{~d}$ & 0,0 & $\mathrm{c}^{1}$ & 0,0 & $\mathrm{c}$ & 0,0 & $\mathrm{c}$ \\
Testemunha suja & $0,0 \mathrm{~d}$ & 0,0 & $\mathrm{c}$ & 0,0 & $\mathrm{c}$ & 0,0 & $\mathrm{c}$ \\
S-metolachlor & $41,25 \mathrm{~b}$ & $38,75 \mathrm{~b}$ & $37,50 \mathrm{~b}$ & $41,25 \mathrm{~b}$ \\
Clomazone & $65,0 \mathrm{a}$ & $71,25 \mathrm{a}$ & $75,0 \mathrm{a}$ & $72,50 \mathrm{a}$ \\
Diclosulam & $26,25 \mathrm{c}$ & $22,50 \mathrm{~b}$ & $28,75 \mathrm{bc}$ & $26,25 \mathrm{bc}$ \\
Sulfentrazone & $30,0 \mathrm{bc}$ & $32,50 \mathrm{~b}$ & $26,25 \mathrm{bc}$ & $28,75 \mathrm{~b}$ \\
Pendimenthalin & $63,75 \mathrm{a}$ & $84,50 \mathrm{a}$ & $80,50 \mathrm{a}$ & $88,75 \mathrm{a}$ \\
\hline CV (\%) & 18,56 & 22,66 & 42,04 & 31,85 & \\
\hline
\end{tabular}

Médias com letras minúsculas distintas na coluna diferem pelo teste Tukey $(p \leq 0,05)$.

O clomazone é um herbicida seletivo, utilizado em pré ou pós-emergência inicial no controle de várias plantas daninhas, na cultura do arroz irrigado no Sul do Brasil (SCHREIBER, 2013). É também utilizado em culturas como a soja, mandioca, cana-de-açúcar e algodão. O pendimethalin registrado para a cultura de soja e recomendado no controle de plantas daninhas gramíneas e algumas folhas largas em pré-emergência, preferivelmente logo após a semeadura da cultura ou no prazo máximo de cinco dias após a semeadura (EMBRAPA, 2006).

O diclosulam é absorvido principalmente pelas raízes e caulículo, possui ação sistêmica e tem metabolismo rápido em espécies tolerantes como soja. Os sintomas, que se tornam evidentes uma a duas semanas após a aplicação, incluem paralisação do crescimento, amarelecimento dos meristemas e redução do sistema radicular, com as raízes secundárias apresentando-se uniformemente curtas e engrossadas (EMBRAPA, 2006).

Os mesmos tratamentos (clomazone e pendimenthailin) também foram eficientes para o controle de capim-arroz na cultura da soja, porém o herbicida smetolachlor mostrou-se eficiente no controle de folhas estreitas na cultura (Tabela $5)$.

TABELA 5. Controle de capim-arroz aos 7, 14, 21 e 28 DAE. FAEM/UFPel- Capão do Leão, RS, 2014.

\begin{tabular}{|c|c|c|c|c|c|c|c|c|}
\hline Tratamentos & \multicolumn{8}{|c|}{ Controle Capim-arroz } \\
\hline & \multicolumn{2}{|c|}{$7 \mathrm{DAE}$} & \multicolumn{2}{|c|}{$14 \mathrm{DAE}$} & \multicolumn{2}{|c|}{$21 \mathrm{DAE}$} & \multicolumn{2}{|c|}{$28 \mathrm{DAE}$} \\
\hline Testemunha limpa & 0,0 & $\mathrm{~d}$ & 0,0 & $d^{1}$ & 0,0 & $d$ & 0,0 & $\mathrm{C}$ \\
\hline Testemunha suja & 0,0 & $d$ & 0,0 & $d$ & 0,0 & $d$ & 0,0 & $\mathrm{C}$ \\
\hline S-metolachlor & 98,0 & a & 98,00 & a & 94,25 & a & 98,0 & a \\
\hline Clomazone & 97,50 & a & 97,50 & a & 95,25 & $\mathrm{a}$ & 98,0 & a \\
\hline Diclosulam & 30,0 & C & 30,0 & C & 17,50 & $\mathrm{C}$ & 25,0 & $\mathrm{~b}$ \\
\hline Sulfentrazone & 46,25 & $b$ & 46,25 & $b$ & 27,50 & $b$ & 26,25 & b \\
\hline Pendimenthalin & 97,0 & a & 97,00 & a & 95,00 & $\mathrm{a}$ & 98,50 & a \\
\hline $\mathrm{CV}(\%)$ & 4,80 & & 4,82 & & 7,96 & & 7,25 & \\
\hline
\end{tabular}

${ }^{1}$ Médias com letras minúsculas distintas na coluna diferem pelo teste Tukey $(p \leq 0,05)$.

Houve diferenças entre os tratamentos herbicidas para o controle de capimarroz na cultura da soja (Tabela 5). Aos 7, 14, 21 e 28 DAE o controle mais eficiente 
da planta daninha na soja, foi quando submetidas aos tratamentos com os herbicidas clomazone, pendimenthalin e s-metolachlor onde não diferiram entre si nos dias avaliados.

Aos 7, 14 e 21 dias após a emergência, o herbicida sulfentrazone mostrou-se mais eficiente no controle do capim-arroz comparado ao herbicida diclosulam (Tabela 5). Aos 28 DAE os tratamentos com sulfentrazone e diclosulam não diferem estatisticamente entre si. Avaliando a eficácia do herbicida diclosulam, em diferentes doses no controle de duas espécies daninhas (Ipomoea grandifolia e Sida rhombifolia) foram observados elevados níveis de controle do herbicida diclosulam a partir da dose de 21,8 g i.a. ha-1 quando aplicado diretamente sobre o solo (CARBONARI, 2008). Fato não observado no presente trabalho com as plantas daninhas utilizadas.

Não se observou diferença estatística para a massa da matéria seca da parte aérea (MMSPA) da soja entre os tratamentos (Tabela 6). No entanto para o nabo a menor produção de MMSPA foi obtida nos tratamentos com pendimethalin e clomazone os quais não diferiam da testemunha limpa, mostrando-se eficientes no controle de nabo, quando comparados aos demais tratamentos que apresentaram uma produção de massa seca superior. Para a planta daninha capim-arroz os herbicidas s-metolachlor, clomazone e pendimethalin foram eficientes no controle não havendo capim-arroz na avaliação aos 28 DAE para que pudesse ser determinado a massa seca, sendo estes eficientes e equivalendo-se a testemunha limpa. No entanto o sulfentrazone não apresentou controle satisfatório de tal forma que a produção de massa seca de capim-arroz não diferiu estatisticamente da testemunha suja.

TABELA 6. Massa da matéria seca da parte aérea (MMSPA) de soja, naboforrageiro e capim-arroz aos 28 DAE. FAEM/UFPel- Capão do Leão, RS, 2014.

\begin{tabular}{|c|c|c|c|c|c|}
\hline \multirow[t]{2}{*}{ Tratamentos } & \multicolumn{5}{|c|}{ MMSPA (g planta ${ }^{-1}$ ) } \\
\hline & Soja & \multicolumn{2}{|c|}{ Nabo-forrageiro } & \multicolumn{2}{|c|}{ Capim-arroz } \\
\hline Testemunha limpa & 1,0 & 0,0 & $b^{1}$ & 0,0 & $\begin{array}{ll}\mathrm{C} \\
\end{array}$ \\
\hline Testemunha suja & 0,78 & 3,60 & a & 2,62 & a \\
\hline S-metolachlor & 0,59 & 3,46 & a & 0,0 & C \\
\hline Clomazone & 0,86 & 1,10 & b & 0,0 & C \\
\hline Diclosulam & 0,32 & 3,12 & a & 1,84 & $\mathrm{~b}$ \\
\hline Sulfentrazone & 0,40 & 3,14 & a & 2,30 & $a b$ \\
\hline Pendimethalin & 0,75 & 0,62 & $b$ & 0,0 & $\mathrm{C}$ \\
\hline CV (\%) & 51,07 & 28,97 & & 30,5 & \\
\hline
\end{tabular}

Médias com letras minúsculas distintas na coluna diferem pelo teste Tukey $(p \leq 0,05)$.

${ }^{\text {ns }}$ Não significativo.

\section{CONCLUSÕES}

Os herbicidas pré-emergentes diclosulam e sulfentrazone apresentaram a maior fitotoxicidade na soja aos 28 DAE. O melhor controle de nabo foi obtido com o pendimethalin e clomazone e para o capim-arroz o melhor controle observado foi também para os referidos herbicidas acrescendo s-metolachlor.

O herbicida sulfentrazone apresentou o menor controle das plantas daninhas e maior fitotoxicidade na soja. 


\section{AGRADECIMENTOS}

Ao Prof. Dr. José Alberto Noldin pelo auxílio e disponibilidade na execução desse trabalho.

\section{REFERÊNCIAS}

CARBONARI, C. A., MESCHEDE, D. K.; CORREA, M. R., VELINI, E. D.; TOFOLI, G. R. Eficácia do herbicida diclosulam em associação com a palha de sorgo no controle de Ipomoea grandifolia e Sida rhombifolia. Planta Daninha, v. 26, n. 3, p. 657-664, 2008.

CORREIA, N.M., DURIGAN, J.C. Controle de plantas daninhas na cultura de soja resistente ao glifosato. Bragantia, v. 69, p.319-327, 2010.

DAN, H. A; BARROSO, A. L. L; DAN, L. G. M; FINOTTI, T. M; FELDKIRCHER, C; SANTOS, V. S; Controle de plantas daninhas na cultura do milho por meio de herbicidas aplicados em pré-emergência. Pesquisa Agropecuária Tropical, v. 40, n. 4, p. 388-393, 2010.

EMBRAPA - Empresa Brasileira de Pesquisa Agropecuária. Principais herbicidas recomendados para cultura de soja no preparo convencional e no sistema plantio direto. (Documentos Online). Passo Fundo, 2006. Disponível em: < http://www.cnpt.embrapa.br/biblio/do/p_do62_17.htm>

MONQUERO, P. A., SILVA, P. V., SILVA HIRATA, A. C., TABLAS, D. C., ORZARI, I. Lixiviação e persistência dos herbicidas sulfentrazone e imazapic. Planta Daninha, v. 28, n. 1, p. 185-195, 2010.

SCHREIBER, F, de AVILA, L.A., SCHERNER, A., MOURA, D.S., HELGUEIRA, D.B. Plants sensitive to clomazone in vapor phase. Ciência Rural, v. 43, n. 10, p. 18171823, 2013.

VARGAS, L.; DE OLIVEIRA, O.; LORIS P. Manejo de plantas daninhas em fruticultura sob sistema de produção convencional, integrada e orgânica. 2013. Disponível em: < http://www.cnpuv.embrapa.br/tecnologias/pin/pdf/p_14.pdf>

VELHO, G. F., CRUSCIOL, C.A.C., VELINI, E.D., CASTRO, G.S.A., BORGHI, E. Weed competition between Brachiaria plantaginea and rice culture, cv.'Primavera'. Planta Daninha, v. 30, n. 1, p. 17-26, 2012.

VIDAL, R.A.; MEROTTO JR., Capítulo 10 - Herbicidas Inibidores de Fotossistema 2. In: VIDAL, R. A.; MEROTTO Jr., Herbicidologia.1.ed. Porto Alegre, 2001.

WEBER, B. J.; WILKERSON, G. G.; REINHARDT, C. F. Calculating pesticide sorption coefficients $(\mathrm{Kd})$ using selected soil properties. Chemosphere, v. 55, n. 2, p. 157-166, 2004. Disponível em: < doi:10.1016/j.chemosphere.2003.10.049> 